\title{
Flow Development through a Duct and a Diffuser Using CFD
}

\author{
Prasanta K.Sinha ${ }^{1}$, A.K.Biswas ${ }^{2}$, A.N. Mullick ${ }^{2}$ and B.Majumdar ${ }^{3}$ \\ ${ }^{1}$ Durgapur Institute of Advanced Technology\& Management, West Bengal, India \\ ${ }^{2}$ Department of Mechanical Engineering, National Institute Technology, West Bengal, India \\ ${ }^{3}$ Department of Mechanical Engineering, Jadavpur University, Kolkata, West Bengal, India
}

\begin{abstract}
In the present paper an extensive study of rectangular cross-sectioned C-duct and C-diffuser is made by the help of 2-D mean velocity contours. Study of flow characteristics through constant area duct is a fundamental research area of basic fluid mechanics since the concepts of potential flow and frictional losses in conduit flow were established. C-ducts are used in aircraft intakes, combustors, internal cooling systems of gas turbines, ventilation ducts, wind tunnels etc., while diffuser is mechanical device usually made in the form of a gradual conical expander intended to raise the static pressure of the fluid flowing through it. Flow through curved ducts is more complex compared to straight duct due to the curvature of the duct axis and centrifugal forces are induced on the flowing fluid resulting in the development of secondary motion (normal to the primary flow direction) which is manifested in the form of a pair of contra-rotating vortices. For a diffuser in addition to the secondary flow, the diverging flow passage, which causes an adverse stream wise pressure gradient, can lead to flow separation. The combined effect may result $\mathrm{n}$ non uniformity of total pressure and total pressure loss at the exit. A comparative study of different turbulent models available in the Fluent using $y^{+}$as guidance in selecting the appropriate grid configuration and turbulence models are done. Standard k- $\varepsilon$ model and RSM models are used to solve the closure problem for both the constant area duct and the diffuser. It has been observed that the Standard k-e model predicts the flow through the constant area duct and the diffuser within a reasonable domain of the $y^{+}$range.
\end{abstract}

Keywords: C-duct, C-diffuser, Secondary Motion, Wall $y^{+}$Strategy, k- $\varepsilon$ model, Fluent solver

Nome nclature
\begin{tabular}{|l|l|}
\hline Dn & Dean Number \\
\hline $\mathrm{L}$ & Centerline length \\
\hline $\mathrm{R}_{\mathrm{c}}$ & Mean Radius of curvature \\
\hline $\mathrm{R}_{\mathrm{e}}$ & Reynold's number $\left(\mathrm{U}_{\mathrm{av}} \mathrm{D} / \mathrm{v}\right)$ \\
\hline $\mathrm{U}_{\mathrm{av}}$ & Inlet Average Velocity \\
\hline $\mathrm{W}$ & Inlet Width \\
\hline $\mathrm{b}$ & Height of duct \\
\hline$\Delta \beta$ & Angle of turn of the curvature \\
\hline$v$ & Kinematic Viscosity \\
\hline
\end{tabular}

\section{INTRODUCTION}

Diffuser is a mechanical device usually made in the form of a gradual conical expander intended to raise the static pressure of the fluid flowing through it. Specific use of a diffuser with a proper area ratio should also ensure an adequate amount of uniform flow at the outlet together with a considerable static pressure. Diffusers play an important role in many engineering and industrial applications to accomplish the objectives under geometric constraints. In aircraft gas turbines, high velocity air from the wing or fuselage first flows through a diffusing intake where it is decelerated for increasing the pressure, and then fed to the engine compressor. In the design of ventilation and air conditioning systems, diffusers are commonly used for discharging the conditioned air into the space to be cooled in order to reduce the air velocity and increase the static pressure. In these applications, on one hand there is restriction on space as well as design compatibility to match with the shortest possible duct length, while on the other hand, given cross-sectional shapes at inlet and outlet of the duct has to be satisfied; this compelled to the use of curved diffusers. C-ducts are used in aircraft intakes, combustors, internal cooling system of gas turbines, ventilation ducts, wind tunnels etc. Heat exchangers in the form of curved ducts are used widely in food processing, refrigeration and hydrocarbon industries. Gas turbine engine components such as turbine compressors, nozzle etc. utilise several complex duct configuration. Performance of duct flow depends upon the geometrical and dynamical parameters of the duct. So it is very much essential to design the duct with proper geometry to imp rove the performance.

Study of flow characteristics through constant area ducts is a fundamental research area of basic fluid mechanics since the concepts of potential flow and frictional losses in conduit flow were established. Duct is a part and parcel of any fluid- 
mechanical system. It is a passageway made of sheet metal or other suitable material used for conveying air or other gases or liquids at different pressures. Depending on its application the shape the duct may be either of straight, curved, annular, polar, sector, trapezoidal, rhombic etc. Flow through curved ducts has practical importance in chemical and mechanical industries in particular. Obviously, compared to a straight duct, flow in a curved duct is more complex due to curvature of the duct axis. It induces centrifugal forces on the flowing fluid resulting in the development of a secondary motion (normal to primary direction of flow) which is manifested in the form of a pair of counter-rotating vortices. Depending on the objective, fluid mechanical systems often demands for the design of ducts with complex geometry (like inlets, nozzles, diffusers, contractions, elbows etc) albeit with high efficiency. In these applications, design of the ducts is based on the mathematical formulation of the flow field for the prescribed condition.

Flow development in the curved diffusers is a complicate process influenced by different geometrical parameters like $2 \theta, \Delta \beta, \mathrm{AR}, \mathrm{AS}$, centreline shape etc. as well as the dynamical parameters like inlet Mach number, in let turbulence etc. In any internal flow, fluid near the flow axis moves at higher velocity than the fluid near the walls due to the influence of boundary layer. In a curved diffuser, due to the presence of centreline curvature, fluid near the flow axis is acted upon by a larger centrifugal force than fluid near the walls. This centrifugal pressure difference (transverse pressure gradient) forces the faster moving fluid to move outwards pushing the fluid in the boundary layer at the outer wall around the sides towards the inner wall; thus a significant secondary flow (normal to the primary flow direction) is produced. In addition, the diverging flow passage, which causes an adverse stream wise pressure gradient, can lead to flow separation. The combined effect may result in nonuniformity of total pressure and total pressure loss at diffuser exit.

Probably, Fox \& Kline, 1962, carried out the first systematic studies on 2-D curved diffusers. They analyzed the performance of the diffusers by varying the ratio between the centreline length to inlet diameter and also varying the angle of turn. They concluded that a stall occurred at an angle equal to or greater than $50^{\circ}$. Shimizu et al., 1986 experimentally studied the performance and internal flow through twisted Sshaped (coiled), U shaped, and snake shaped bend diffusers having circular cross-section and different area ratios (1.3 to 15.9$)$, divergent angles $\left(3^{\circ}, 6^{\circ}\right)$, and curvature ratios $(3,6)$, which they have described in their 1st and 2nd report. They found that the generated separation in the upstream portion of the diffuser was detrimental to the performance of the diffusers, which could be overcome by varying the inlet velocity distribution and proper selection of an optimum combination of the divergent angle and the radius to curvature ratio. Shimizu et al., 1992 experimentally investigated the influence of aspect ratios ( 0.25 to 8 ) on the performance and generation of secondary flow in sinuous conduits of rectangular crosssection. They observed that the hydraulic losses decrease with increase in aspect ratio, and in case of smallest aspect ratio flow separation occurred in downstream sections resulting in large hydraulic losses. The number of secondary vortex pairs was found with the increase in aspect ratio (1 to 4 to 8 ). Dominy and Kirkham, 1996, made extensive analysis of the influence of wakes on the flow through diffusing $\mathrm{S}$ shaped annular duct. Based on the inlet velocity distribution (with wakes and without wakes) they found that the simple axial wakes strongly influence the flow, by affecting not only the hub and casing boundary layers but also more significantly the strong secondary flows, resulting in total pressure distortions. Majumdar et al., 1988 experimentally studied flow through a high aspect ratio $(\mathrm{AS}=6), \mathrm{AR}=2, \Delta \beta=90^{\circ}$, centreline length $0.6 \mathrm{~m}$, and $\mathrm{Rc}=0.382 \mathrm{~m}$ curved diffuser. They observed that the streamwise bulk flow shifted towards the outside vertical wall, which was mainly due to the centrifugal effect arising out of the centreline curvature. They studied wall pressure distribution and found that the wall static pressure increased continuously on both convex and concave walls due to diffusion and showed a pressure recovery close to $50 \%$. They also observed that there was no separation in the diffuser except for a small pocket of separation on the convex wall just at the exit of the diffuser. Counter rotating vortices were detected from 300 turn of the diffuser. Anand et al., 2003 experimentally investigated the influence of the turning angle $\left(15^{\circ} / 15^{\circ}, 22.5^{\circ} / 22.5^{\circ}\right.$, and $\left.30^{\circ} / 30^{\circ}\right)$ on the performance characteristics and the structure of the flow through a long S- shaped circular diffuser. Velocity, static pressure and total pressure distributions were measured at different planes along the length of the diffuser using a five hole impact probe and the turbulence intensity distribution at those planes were measured using a normal hot-wire probe. They found that the static pressure recovery coefficients of these test diffusers decreased with increase in the turning angle and were to be always lower than the ideal value of 0.72 . The low performance is attributed to the generation of secondary flows due to geometric curvature and additional losses 
as a result of high surface roughness $(-0.5 \mathrm{~mm})$ of the diffusers. The pressure recovery coefficients of these circular test diffusers were comparatively lower than that of an S- shaped rectangular diffuser of nearly the same area ratio, even with a larger turning angle $\left(90^{\circ} / 90^{\circ},\right)$. The total pressure loss coefficient for all the diffusers were nearly the same and seemed to be independent of the angle of turn. The flow distribution was found to be more uniform at the exit for diffusers with higher angle of turn. ElAskary and Nasr, 2009 studied turbulent flow through a combined bend-diffuser system, consisting of a $90^{\circ}$ bend followed by a diffuser of rectangular cross-section and different angles $(2 \theta)$ ranging from $6^{\circ}-30^{\circ}$ at different inflow Reynolds numbers. Experimental and simulated results showed that, depending upon the inlet Reynolds number there is an optimum diffuser angle, which produces minimum pressure loss and hence good performance of such complex geometry. The asymmetry of flow in the diffuser due to the installation of a bend at the upstream generates a side load to the diffuser wall, which increases with increas ing diffuser angle and Reynolds number.

Rowe, 1970, carried out experiments on circular $90^{\circ}$ and $180^{\circ}$ turn curved ducts with $\mathrm{Re}=0.4 \times 10^{5}$ and reported the generation of contra rotating vortices with in the bends. Bansod \& Bradshaw, 1972, studied the flow characteristics with in the $22.5^{\circ} / 22.5^{\circ} \mathrm{S}$ shaped constant area ducts of different lengths and radii of curvature. They reported the development of a pair of contrarotating vortices in the low pressure zone at the exit of the duct which was the consequence of the effect of stream wise vortices developed in the first half of the duct. Enayet et al., 1982, investigated the turbulent flow characteristics through $90^{\circ}$ circular curved duct of curvature ratio 2.8. It was observed that the thickness of the in let boundary layer has a significant role on generation of secondary motion with in the duct. Azzola et al., 1986, have studied the turbulent flow characteristics through $180^{\circ}$ circular bend with curvature ratio of 3.375 through experiments as well as computational methods. They observed a pair of contra-rotating vortices arising out of secondary motion in both experimental and numerical studies. Lacovides et al., 1987, reported the flow prediction with in $90^{\circ}$ curved duct using numerical simulations based on the experimental investigation by Taylor et al., 1982. They adopted finite volume approach tu solve the semi-elliptical form of equation for 3-D flow analysis considering the wall function in the region close to the wall. The result shows a good agreement between the experimental and numerical analys is. Thangam and Hur, 1990, studied the secondary flow of an incompressible viscous flnid in a curved rectangular duct by using a finite volume method. They reported that with the increase of Dean Number the secondary flow structure evolves into a double vortex pair for low aspect ratio ducts. They correlated friction factor as a function of the Dean Number and aspect ratio. Kim and Patel, 1994, have investigated on a $90^{\circ}$ curved duct of rectangular cross-section with aspect ratio 6 using five-hole probe and cross-wire hot wire anemometer. They reported the formation of vortices on inner wall due to the pressure driven secondary motion originated in the corner region of curved duct. Investigation on the turbulent boundary layer on the wall of an S-shaped wind tunnel for various Reynolds numbers ranging from $3.0 \times 10^{3}$ to $11 \times 10^{3}$ was carried out by Burns et al., 1999. They used hot wire probe to measure mean velocity and Reynolds stresses. They interpreted their results for turbulence response and evaluated Reynolds Stress Transport Equations. Singh et al., 2004, experimentally studied the flow and performance characteristics of a $\mathrm{Y}$ shaped duct having an aspect ratio 1 and 1.66 for two inlet limbs with angle of turn $90 \% 90^{\circ}$. The average inlet velocities in the two limbs were $29 \mathrm{~m} / \mathrm{s}$ and $24 \mathrm{~m} / \mathrm{s}$ respectively. The longitudinal velocity and static and total pressure were measured by using a 3-hole pressure probe. They observed that the pressure recovery coefficient and loss coefficient increased continuously from inlet to the exit of the diffusing duct and are nearly same.

\section{EXPERIMENTAL FACILITY}

The experiments were conducted in the Aerodynamics Laboratory, National Institute of Technology, Durgapur. The measurements were carried out in an open-circuit wind tunnel (refer to Fig. 1). A centrifugal air blower coupled with a 3Phase, $5.5 \mathrm{~kW}$, and $2870 \mathrm{rpm}$ electric motor delivered air through a diffuser of area ratio 3.65 and of length $1.38 \mathrm{~m}$, into a large settling chamber. A flexible coupling made of canvas, installed in between the blower exit and the diffuser, was used to isolate the settling chamber from transmission of vibration of the blower and driving motor. The rate of mass flow of air through the test rig was controlled by throttling the blower suction with the help of a flap gate mounted on it. The settling chamber, $2.88 \mathrm{~m}$ in length and $0.6 \mathrm{~m}$ in diameter, was provided with a flow passage having a honeycomb structured cross section and three sets of nylon screens at three different locations, to straighten the flow and reduce the level of turbulence. A contraction piece of $0.4 \mathrm{~m}$ length, symmetric about the centre line and made from ply wood was installed in 

ISSN : 2248-9622, Vol. 7, Issue 1, ( Part 4) January 2017, pp.46-54

between the settling chamber and the inlet piece of the curved diffuser, to provide continuity as well as to accelerate the flow. A straight inlet piece, $0.1 \mathrm{~m}$ in length and of cross-section $0.05 \mathrm{~m}$ (WI) X 0.1m (b) was connected in between the contraction piece and the test diffuser. The contraction piece, with high contraction ratio, followed by the straight duct at the upstream of the curved diffuser ensured uniform flow to the test diffuser. Another constant area straight duct of cross-section $0.1 \mathrm{~m} \mathrm{X} 0.1 \mathrm{~m}$ and $0.1 \mathrm{~m}$ in length was placed at the downstream of the test diffuser to facilitate a continuous flow from the diffuser outlet so as to minimize the effect of the downstream atmosphere. Details of the test diffuser along with the in let and outlet pieces are shown in Fig.2. The test diffuser was fabricated from transparent Perspex sheets. The crosssectional area was increased by linearly varying the width from $0.05 \mathrm{~m}$ at in let to $0.1 \mathrm{~m}$ at the exit, over the total centre line length of $0.6 \mathrm{~m}$ while the height was kept constant at $0.1 \mathrm{~m}$. The width was equally distributed normal to the centerline. The curved diffuser was fabricated in four segments each having a $22.5^{\circ}$. So angle of turn and radius of curvature $0.382 \mathrm{~m}$. For wall static pressure measurements, the diffusers together with the in let and out let pieces were divided in six sections (Inlet, A, B, C, D, and Outlet) as shown in Fig.2. Stainless steel tubes of $2 \mathrm{~mm} \mathrm{OD}$ and $1 \mathrm{~mm}$ ID were inserted and glued into the $2 \mathrm{~mm}$ drilled holes at all chosen measuring stations. Initially all the pressure taps were plugged by caps. For measurement of static pressure at a particular station the cap on the steel tube was replaced by a long transparent polythene tube which was connected to one of the limbs of a multi-tube inclined differential manometer (inclined at 35" with the vertical) and other limbs were kept open to a1mosphere. Kerosene oil was chosen as manometric liquid.

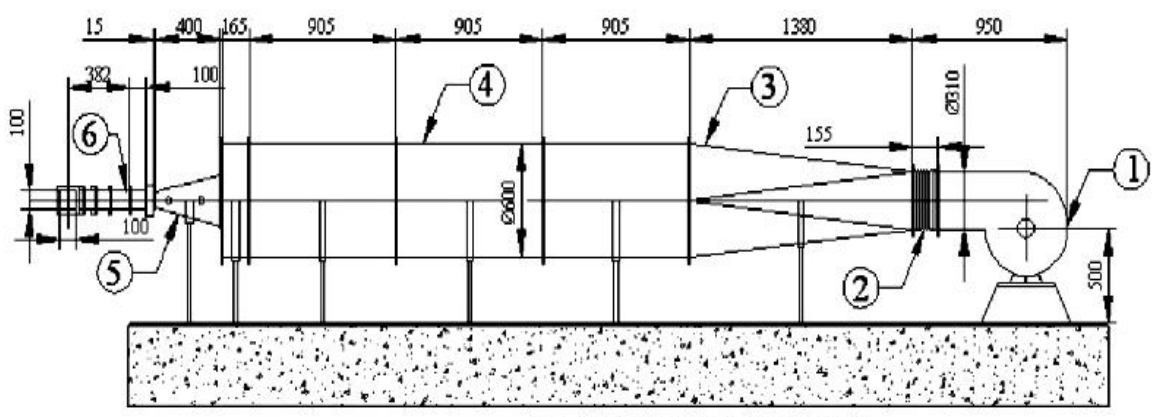

All dimensions are in $\mathrm{mm}$.

\section{(1) Centrifugal Blower (2) Flexible Coupling (3) Diffuser (4) Settling Chamber (5) Contraction Piece (6) Test Diffuser}

Fig. 1. Schematic Layout of the Experimental Set-up

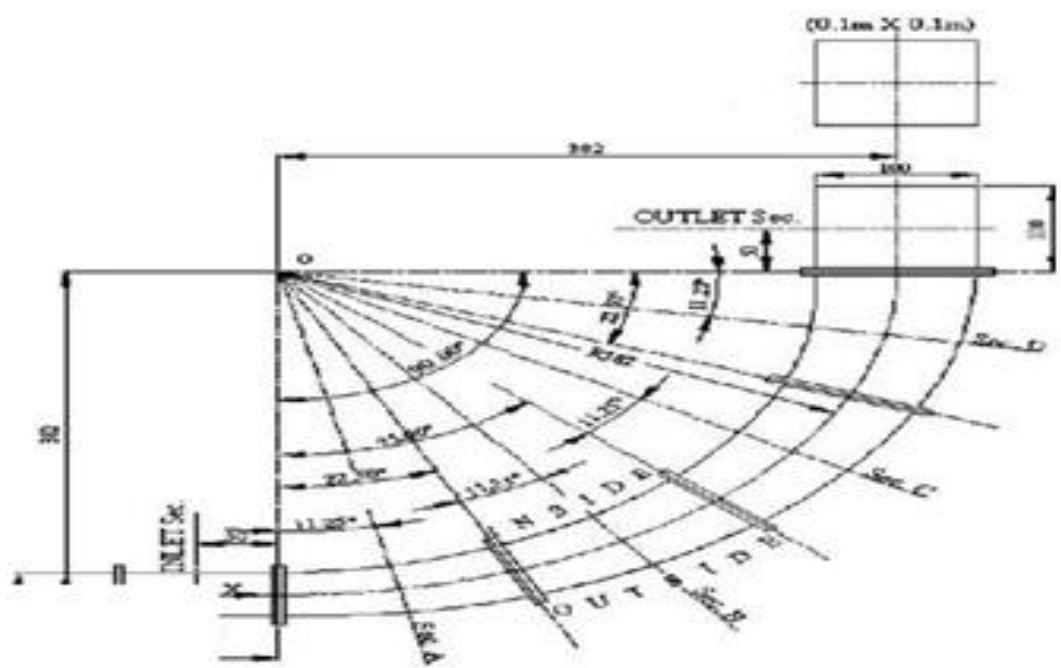

Fig.2. Geometry ofCurved Diffuser and Measuring Locations

The complete geometry of curved duct under a test is shown in fig. 3 . It is a rectangular $90^{\circ}$ curved duct with $50 \mathrm{~mm}(\mathrm{~W})$ and height $100 \mathrm{~mm}$ with centre line length of $600 \mathrm{~mm}(\mathrm{~L})$. It is constituted of four equal segments each subtended an angle of $22.5^{\circ}$. The parallel horizental wall (top and bottom) of the duct are made from $12 \mathrm{~mm}$ thick transparent Perspex sheet whereas the curved vertical walls 
(convex and concave) are fabricated with $3 \mathrm{~mm}$ thick perplex sheet. These side walls of the ducts are made by bending the sheet and fastened by screws with the top and bottom parallel walls. The rad ii of curvature of outer and inner curved walls of duct of radius $407 \mathrm{~mm}$ and $357 \mathrm{~mm}$ respectively. The mean radius of the curvature of the duct is $382 \mathrm{~mm}$ (Rc). Two straight constant area ducts of cross sectional area
$50 \mathrm{~mm} \times 50 \mathrm{~mm}$ were attached as extension pieces at the inlet and exit respectively. The length of the two extension pieces are $100 \mathrm{~mm}$. They help fixing the inlet and outlet condition of the flow. There are six sections considered at the middle point of these six pieces of rectangular curved duct. These sections are inlet-section, section-A, section-B, section-C, section-D and outlet-section as shown in Fig.3.

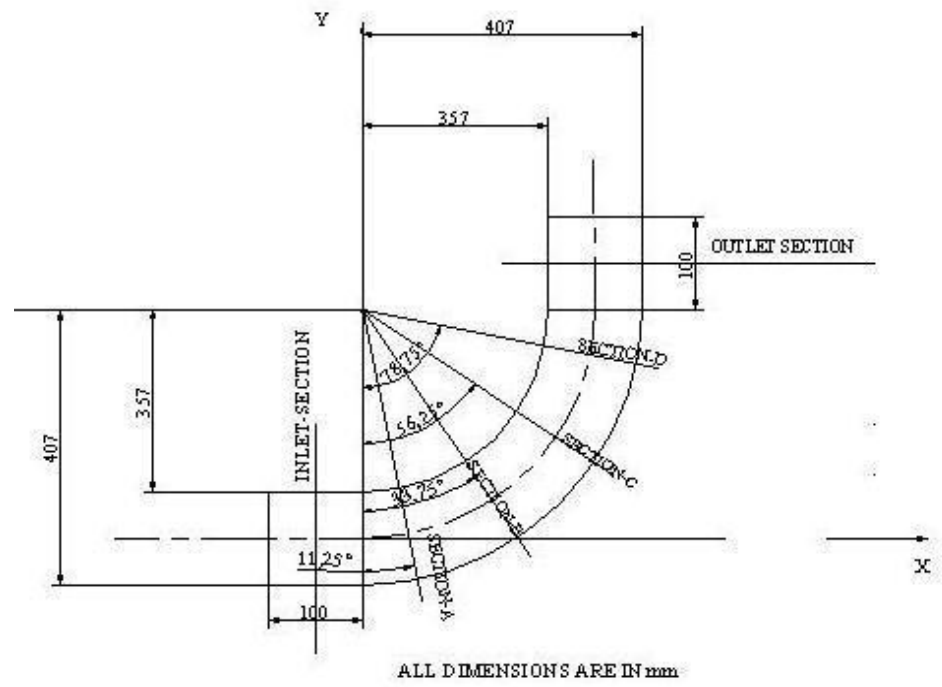

Fig.3. Geometry of $90^{\circ}$ Curved Duct and Measuring Locations

\section{RESULTS AND DISCUSSION}

Flow development in the curved flow passage is a complicate due the influenced by different geometrical parameters like total angle of divergence $(2 \theta)$, angle of turn $(\Delta \beta)$, area ratio (AR), inlet aspect ratio (AS), centreline shape etc. as well as the dynamical parameters like in let Mach number, inlet turbulence etc. In the present study, experimental investigations have been carried out on curved diffusers to enable a comparative study of the flow development and performance characteristics for different centre line shapes and different inlet mass averaged velocities. The straight inlet duct, between the contraction section and the test pieces under investigation, was short $(2 \mathrm{Wl})$ and the Inlet Section was located at its mid-length. The flow at the inlet was mostly fully developed with a uniform velocity distribution throughout. At the inlet the mass average velocity is maintained at $40 \mathrm{~m} / \mathrm{s}$ in both the cases. It is evident that there develops a velocity gradient in the trans verse direction forboth the $\mathrm{C}$-shaped constant area duct and C-shaped diffuser which is mainly due to the influenced of the centrifugal action of the centreline curvature and also the same is attenuated in the case of $\mathrm{C}$-shaped diffuser due to the adverse pressure gradient. The Fig. 4 depicts the distribution of mean velocity in the formof2-Dcontours.
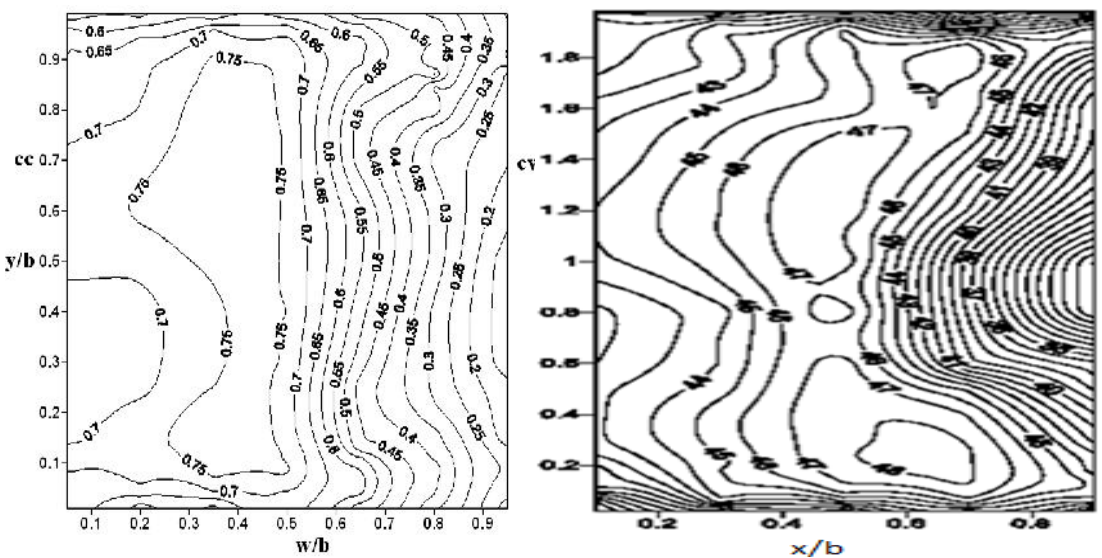

Fig.4. Distribution of mean velocity contour 


\subsection{Wall $y^{+}$Strategy}

The wall $y^{+}$is a non-dimensional number similar to local Reynolds number, determining whether the influences in the wall-adjacent cells are la minar or turbulent, hence indicating the part of the turbulent boundary layer that they resolve.

$$
y^{+}=\frac{u_{\text {zair }}}{v_{\text {air }}} y
$$

The wall $y^{+}$is a non-dimensional number similar to local Reynolds number, determining whether the influences in the wall-adjacent cells are laminar or turbulent, hence indicating the part of the turbulent boundary layer that they resolve.

$$
y^{+}=\frac{u_{\text {zair }}}{v_{\text {air }}} y
$$

The subdivisions of the near-wall region in a turbulent boundary layer can be summarized as follows (Fluent,2006), [16]:

1. $y^{+}<5$ : in the viscous sub layer region (velocity profiles is assumed to be laminar and viscous stress dominates the wallshear)

2. $5<y^{+}<30$ : buffer region(both viscous and turbulent sheardominates)

3. $30<y^{+}<300$ : Fully turbulent portion or log-law region (corresponds to the region where turbulent shear predominates

The wall $y^{+}$is a non-dimensional distance similar to local Reynolds number, often used in CFD to describe how co arse or fine a mesh is for a particular flow. It is the ratio between the turbulent and laminar influences in acell. Very close to the wall, viscous damping reduces the tangential velocity fluctuations, while kinematics blocking reduces the normal fluctuations. Towards the outer part of the near-wall region, however, the turbulence is rapidly augmented by the production of turbulent kinetic energy due to the large gradients in mean velocity. Accurate presentation of the flow in the nearwall region determines successful prediction of wallbounded turbulent flows. Values of $y^{+}$close to the lower bound $\left(y^{+} \approx 30\right)$ are most desirable for wall functions whereas $y^{+}=1$ are most desirable for nearwall modelling.

\subsection{Computational methodology and boundary layer condition for $C$ duct}

The pre processor GAMBIT is used to create the geometry defining the problem and descrize the domain while FLUENT 6.9 is employed to descrize and solve the governing equations. The generated mesh size was $10^{5}$ cells. Three boundary conditions are specified, i.e. velocity inlet, pressure outlet and wall. No slip boundary conditions were applied to the walls of the duct. For velocity in let boundary condition the turbulent intensity was taken as $10 \%$ and the hydraulic diameter was taken as $66.67 \mathrm{~mm}$ where as for pressure outlet boundry condition the backflow modified turbulent viscosity was taken as $0.001 \mathrm{~m}^{2} / \mathrm{s}$.

\begin{tabular}{|c|c|c|}
\hline Table 1 \\
\hline $\begin{array}{c}Y^{+} \\
\text {Tmin im um })\end{array}$ & $\begin{array}{c}Y^{+} \\
(\text {maxim um }\end{array}$ \\
\hline Standard K-epsilon & 24.41 & 160.2977 \\
\hline K-Omega & 24.75 & 154.8093 \\
\hline RSM & 20.61 & 162.16 \\
\hline
\end{tabular}

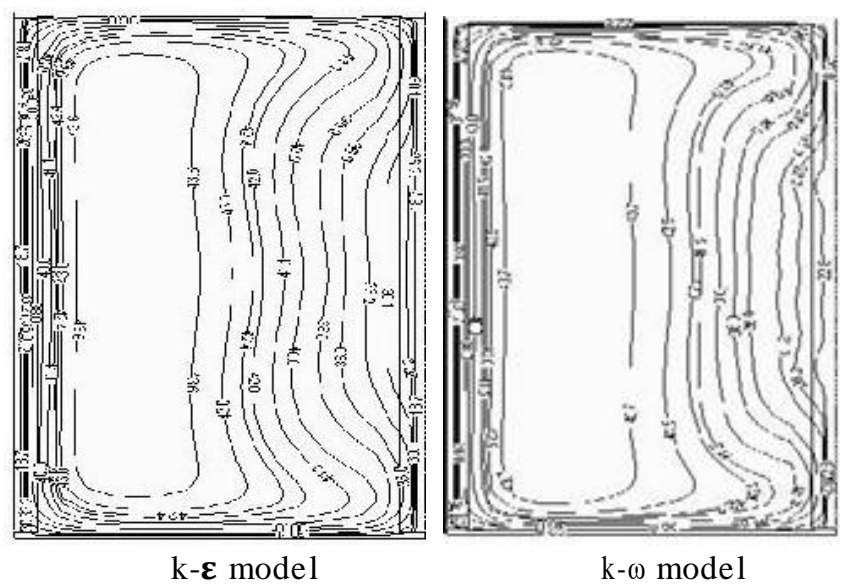




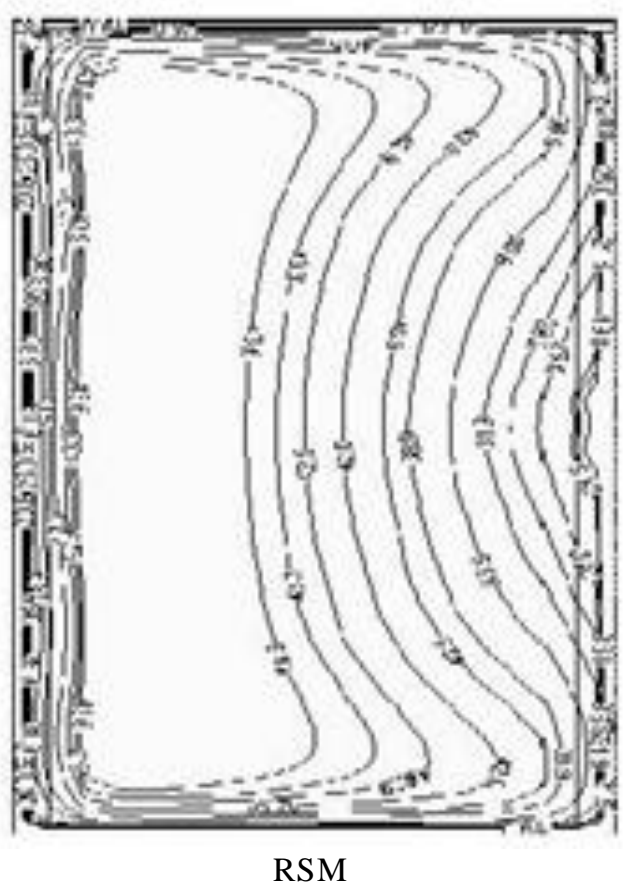

Fig.5: Mean velocity contours at the outlet section of the Cduct

\subsection{Computational methodology and boundary layer condition for $C$ diffuser}

The pre-process or GAMBIT is used to create the geometry defining the problem and discretize the domain while FLUENT 6.9 is employed to discretize and solve the governing equations. Initially the mesh size of 388311 cells was created Three boundary conditions where specified, i.e. velocity inlet, pressure outlet and wall. No slip boundry conditions were applied to the walls of the duct. During the creating of the mesh boundary layer was taken under consideration and the mesh near the walls of the diffuser was dense in comparison with the other region of the diffuser. A turbulence intensity of $6 \%$ and a hydraulic diameter of 0.0666 were taken for inlet and outlet boundary conditions. The standard $\mathrm{k}-\varepsilon$, standard $\mathrm{k}-(\omega)$ and RSM turbulence models where used and the $y^{+}$values were calculated correspondingly. For the case of standard k- $\varepsilon$ turbulence model the mesh was adapted according to the Y-plus value. A general method fordetermin ing the most appropriate mesh configuration is grid independence test, where different meshes are tested until the solution is independent of further refinement, by matching the results to experimental results. But in the present case the method of solution-adaptive refinement was used, where one can add cells where they are needed in the mesh thus enabling the features of the flow field to be better resolved. When adaption is used properly, the resulting mesh is optimal for the solution because the solution is used to determine where more cells need to be added. Here Y-plus adaption have been provided to appropriately refine orcoarsen the mesh along the wall during thesolution process. The minimumallowed $y^{+}$ value of 2 and maximum allowed $y^{+}$was taken to refine the mesh near the wall region. After adapting the grid the mesh size increased to 647031 cells. In compressible, 3D steady flow RANS equations implemented to solve the problem. SIMPLE algorith $\mathrm{m}$ was used for the pressure velocity coupling and QUICK scheme was used to discretize the momentum and turbulence equations. Convergence criterion of 0.001 was taken to get a converged solution. The wall $Y^{+}$values for the different turbulence models considered areasfollows:

Table 2

\begin{tabular}{|l|l|l|}
\hline $\begin{array}{l}\text { Turbulence } \\
\text { Model }\end{array}$ & $\begin{array}{l}Y^{+} \\
\text {(minimum) }\end{array}$ & $\begin{array}{l}Y^{+} \\
\text {(maximum) }\end{array}$ \\
\hline Standard K-epsilon & 3.65 & 61.8 \\
\hline RNG k-epsilon & 4.61 & 105.65 \\
\hline RSM & 4.15 & 102.08 \\
\hline
\end{tabular}




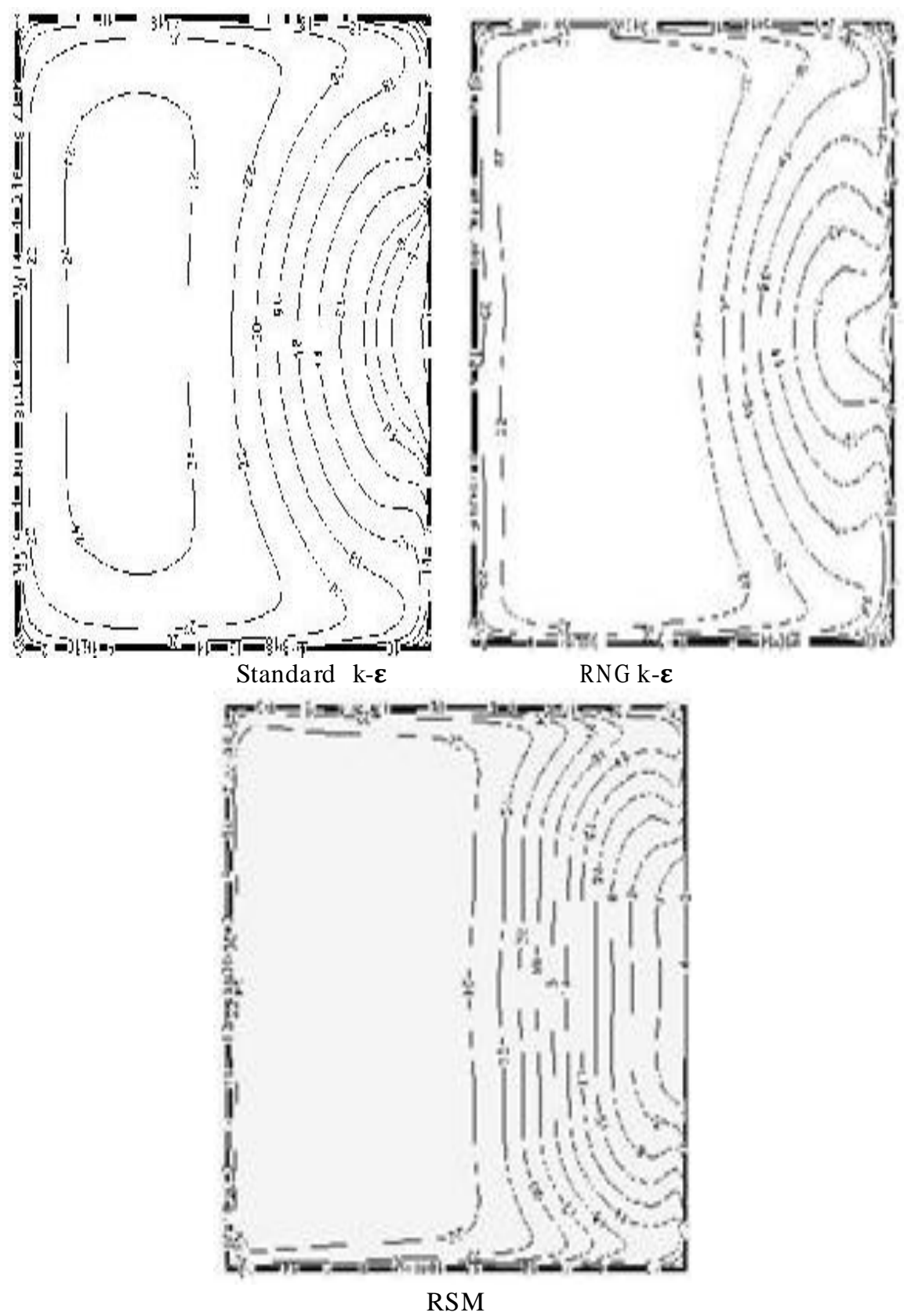

Fig.6 : Mean velocity contours at the outlet section of the Cdiffusing duct

From both experimental and numerical results it can be observed that RSM model predicts the flow more efficiently for both the constant area duct and diffuser. Wall $Y^{+}$value at the viscous blending region is lowest and the same at fully turbulent region is highest for the RSM model for the constant area duct, whereas all the three model resolve the viscous sub layer region for C-d iffuser.

\section{CONCLUSION}

From both experimental and numerical results shows that the high momentum fluids shifted towards the outer wall due to the generation of secondary motion for both C-shaped constant area duct and diffusing duct. For constant area duct based on the $y^{+}$parameter RSM turbulence model predicts the flow in a better way and the velocity distribution for the same qualitatively matches with the experimental results. For diffusing duct also RSM turbulence model predicts the better result based on the $y^{+}$parameter and the velocity distribution for the same also qualitatively matches with the experimental. 


\section{REFERENCES}

[1]. R. W. and Kline, S. J., 1962, Flow Regimes in Curved Subsonic Diffusers, Trans ASME, Journal of Bas ic Engin eering, 84, 303-3

[2]. Shimizu,Y., Nagafusa, M., Sugino, K., and Nakamura,F., 1986. Studies on Performance and Internal Flow of Twisted S-Shaped Bend Diffuser-The So-Called Coiled Bend Diffuser: 1st Report, Trans. ASME Journal of Fluids engineering 108 289-296

[3]. Shimizu,Y., Nagafusa, M., Sugino, K., and and Kubota, T., 1986. Studies of UShaped and Snake-Shaped Bend Diffusers2nd Report, Trans. of ASME Journal of Fluids Engineering 108 297303.

[4]. Shimizu, Y., Futaki, Y., and Martin, C.S., 1992. Secondary Flow and Hydraulic Losses With in Sinuous Conduits of Rectangular Cross section Trans. of ASME Journal of Fluids Engineering 114 593-600.

[5]. Majumd ar, B., Mohan, R., Singh, S.N., and Agrawal, D.P., 1988. Experimental Study of Flow in High Aspect Ratio 90 Deg Curved Diffuser Trans. of ASME Journal of Fluids Engineering 120 8389.

[6]. El-As kary, W.A., and Nasr, M., 2009. Performance of a Bend-Diffuser System: Experimental and Numerical Studies, Journal of Computers \& Fluids 38(1) 160- 170 .

[7]. Enayet, M. M., Gibson, M. M., Taylor, A.M. K. P. and Yianneskis, M., 1982. Laser Doppler measurements of Laminar and Turbulent Flow in a Bend, Int. Journal of Heat and Fluid Flow 3 211-217.

[8]. Kim, W. J. and Patel, V. C., 1994 Orig in and Decay of longitudinal Vortices in the development of flow in a curved rectangular duct Trans. ASME, Journal of Flu id Engineering 116(3) 45-52.

[9]. Lacovides, H., Launder, B. E. and Loizou, P. A., 1987. Numerical Computation of Turbulent Flow through a Squared Sectioned $90^{\circ}$ Bend, Int. Journal of Heat and Fluid Flow 8(4) 320-325..

[10]. Rowe, M., 1970. Measurements and computations flow in pipe bends, Journal of Fluid Mechanics 43(4) 771783.

[11]. Singh, N., Singh, S. N. and Seshadri, V., 2004.FlowCharacteristics of an symmetric Y-Shaped Duct Proceedings of 31st National Conference on Fluid Mechanics and Fluid Power, Jadavpur Uuiversity 749-757.

[12]. Azzola, J., Humpbrey, A. C., Iacovides, Hand Launder, B. E., 1986 Developing circular cross-section: Measurement and Computations, Trans. ASME, Journal of Fluid Eng ineering 108 214-221.

[13]. Bansod, P and Bradshaw, P., 1972. The Flow in S-shaped Ducts, Aeronautical Quarterly 23 131-140.

[14]. Burns, J. M., Ferubolz, H. H. and Mankewitz, P. A., 1999. An experimental investigation of a three dimensional turbulent boundary layer in an S-shaped duct, Journal of Fluid Mechanics 393 175-213.

[15]. Taylor, A.M. K. P., Whitelaw J. H. and Yianneskis, M., 1982. Curved Ducts with Strong Secondary Motion: Velocity Measurements of Developing Laminar and Turbulent Flow, Trans. ASME, Journal of Flu id Engineering, I 04 350-358.

[16]. Tbangam, S. and Hur, N., 1990. Laminar secondary flows in curved rectangular ducts Journal of Fluid Mechanics 217 421-440.

[17]. Fluent "User's Guide Fluent 6.3.26" Fluent Incorporated, USA 2006. 